\title{
СТАЦИОНАРНЫЕ ПРОЦЕССЫ В ТРАНСФОРМАТОРНОМ РЕГУЛЯТОРЕ ПЕРЕМЕННОГО ТОКА УДВОЕННОЙ ЧАСТОТЫ
}

B $\left[{ }^{1,2}\right]$ при анализе трансформаторного регулятора тока на базе двухтактного магнитного удвоителя частоты (ДМУЧ) последовательного типа отмечалось, что его существенным недостатком является относительно большая требуемая мощность цепи подмагничивания. В $\left[{ }^{3}\right]$ описывалась схема ДМУЧ с самоподмагничиванием, в которой в идеализированном случае, благодаря включению в выходную цепь вентилей, мощность цепи независимого подмагничивания приближается к нулю и которая служит источником стабилизированного напряжения.

В новой схеме ДМУЧ (рис. 1) [ $\left.{ }^{4}\right]$ сочетаются положительные свойства обеих исходных схем: малая мощность цепи независимого подмагничивания и возможность управления уровнем выходного тока, что имеет большое практическое значение. Поэтому в настоящей работе подробно анализируются стационарные процессы в усовершенствованной схеме. Знание этих процессов позволит более рационально проектировать соответствующие устройства.

Рассмотрим новую схему при ее работе на активную нагрузку при двухфазном напряжении питания: $u_{11}=U_{1 m} \sin \omega t$ и $u_{12}=$ $=U_{1 m} \cos \omega t$, причем $U_{1 m} \leqslant$ $\leqslant 2 \Phi_{s} w_{1} \omega\left(\Phi_{s}-\right.$ магнитный поток насыщения сердечников). Дополнительно предположим, что сердечники имеют прямоугольные характеристики намагничивания, вентили в выходной цепи идеальные и индуктивность дросселя $L$ в выходной цепи настолько велика, что гармониками тока, кратными четырем, можно пренебречь.

При таких условиях в работе ДМУЧ в зависимости от величины сопротивления нагрузки при фиксированном значении тока подмагничивания возможны в принципе следующие состояния

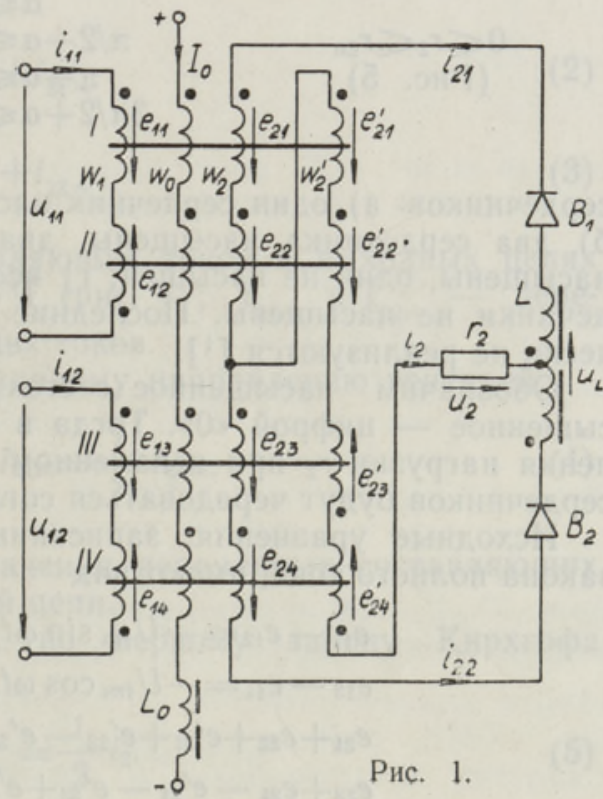


Таблица 1

\begin{tabular}{|c|c|c|c|c|c|}
\hline \multirow[t]{2}{*}{$r_{2}$} & \multirow[t]{2}{*}{ Интервал } & \multicolumn{4}{|c|}{$\begin{array}{c}\text { Номера } \\
\text { сердечников }\end{array}$} \\
\hline & & I & II & III & IV \\
\hline $\begin{array}{l}r_{2} \geqslant r_{2 к р} \\
\text { (Рис. 2) }\end{array}$ & $\begin{aligned} 0 & \leqslant \omega t \leqslant \pi / 2 \\
\pi / 2 & \leqslant \omega t \leqslant \pi \\
\pi & \leqslant \omega t \leqslant 3 \pi / 2 \\
3 \pi / 2 & \leqslant \omega t \leqslant 2 \pi\end{aligned}$ & $\begin{array}{l}0 \\
1 \\
1 \\
0\end{array}$ & $\begin{array}{l}1 \\
0 \\
0 \\
1\end{array}$ & $\begin{array}{l}1 \\
1 \\
0 \\
0\end{array}$ & $\begin{array}{l}0 \\
0 \\
1 \\
1\end{array}$ \\
\hline$r_{2 \kappa p}^{\prime} \leqslant r_{2} \leqslant r_{2 \kappa р}$ & $\begin{array}{c}0 \leqslant \omega t \leqslant \beta \\
\beta \leqslant \omega t \leqslant \gamma \\
\gamma \leqslant \omega t \leqslant \pi / 2 \\
\pi / 2 \leqslant \omega t \leqslant \pi / 2+\beta \\
\pi / 2+\beta \leqslant \omega t \leqslant \pi / 2+\gamma \\
\pi / 2+\gamma \leqslant \omega t \leqslant \pi \\
\pi \leqslant \omega t \leqslant \pi+\beta \\
\pi+\beta \leqslant \omega t \leqslant \pi+\gamma \\
\pi+\gamma \leqslant \omega t \leqslant 3 \pi / 2 \\
3 \pi / 2 \leqslant \omega t \leqslant 3 \pi / 2+\beta \\
3 \pi / 2+\beta \leqslant \omega t \leqslant 3 \pi / 2+\gamma \\
3 \pi / 2+\gamma \leqslant \omega t \leqslant 2 \pi\end{array}$ & $\begin{array}{l}0 \\
0 \\
0 \\
1 \\
1 \\
1 \\
1 \\
0 \\
1 \\
0 \\
0 \\
0\end{array}$ & $\begin{array}{l}1 \\
0 \\
1 \\
0 \\
0 \\
0 \\
0 \\
0 \\
0 \\
1 \\
1 \\
1\end{array}$ & $\begin{array}{l}1 \\
1 \\
1 \\
1 \\
0 \\
1 \\
0 \\
0 \\
0 \\
0 \\
0 \\
0\end{array}$ & $\begin{array}{l}0 \\
0 \\
0 \\
0 \\
0 \\
0 \\
1 \\
1 \\
1 \\
1 \\
0 \\
1\end{array}$ \\
\hline $\begin{array}{c}r_{2 \pi} \leqslant r_{2} \leqslant r_{2 \text { кр }}^{\prime} \\
\text { (Рис. } 4)\end{array}$ & $\begin{array}{c}\alpha \leqslant \omega t \leqslant \beta \\
\beta \leqslant \omega t \leqslant \pi / 2+\alpha \\
\pi / 2+\alpha \leqslant \omega t \leqslant \pi / 2+\beta \\
\pi / 2+\beta \leqslant \omega t \leqslant \pi+\alpha \\
\pi+\alpha \leqslant \omega t \leqslant \pi+\beta \\
\pi+\beta \leqslant \omega t \leqslant 3 \pi / 2+\alpha \\
3 \pi / 2+\alpha \leqslant \omega t \leqslant 3 \pi / 2+\beta \\
3 \pi / 2+\beta \leqslant \omega t \leqslant 2 \pi+\alpha\end{array}$ & $\begin{array}{l}0 \\
0 \\
1 \\
1 \\
1 \\
0 \\
0 \\
0\end{array}$ & $\begin{array}{l}1 \\
0 \\
0 \\
0 \\
0 \\
0 \\
1 \\
1\end{array}$ & $\begin{array}{l}1 \\
1 \\
1 \\
0 \\
0 \\
0 \\
0 \\
0\end{array}$ & $\begin{array}{l}0 \\
0 \\
0 \\
0 \\
1 \\
1 \\
1 \\
0\end{array}$ \\
\hline $\begin{array}{c}0 \leqslant r_{2} \leqslant r_{2 \pi} \\
(\text { Рис. } 5)\end{array}$ & $\begin{array}{c}\alpha \leqslant \omega t \leqslant \pi / 2+\alpha \\
\pi / 2+\alpha \leqslant \omega t \leqslant \pi+\alpha \\
\pi+\alpha \leqslant \omega t \leqslant 3 \pi / 2+\alpha \\
3 \pi / 2+\alpha \leqslant \omega t \leqslant 2 \pi+\alpha\end{array}$ & $\begin{array}{l}0 \\
1 \\
0 \\
0\end{array}$ & $\begin{array}{l}0 \\
0 \\
0 \\
1\end{array}$ & $\begin{array}{l}1 \\
0 \\
0 \\
0\end{array}$ & $\begin{array}{l}0 \\
0 \\
1 \\
0\end{array}$ \\
\hline
\end{tabular}

сердечников: а) один сердечник насыщен, три остальных не насыщены; б) два сердечника насыщены, два не насыщены; в) три сердечника насыщены, один не насыщен; г) все сердечники насыщены; д) все сердечники не насыщены. Последние три режима в этой схеме практически не реализуются $\left[{ }^{1}\right]$.

Обозначим насыщенное состояние сердечника цифрой «1» и ненасыщенное - цифрой «0». Тогда в зависимости от величины сопротивления нагрузки $r_{2}$ при неизменном токе подмагничивания $I_{0}$ состояния сердечников будут чередоваться согласно табл. 1.

Исходные уравнения, записанные на основе законов Кирхгофа и закона полного тока, имеют вид

$$
\begin{aligned}
& e_{11}-e_{12}=-U_{1 m} \sin \omega t, \\
& e_{13}-e_{14}=-U_{1 m} \cos \omega t, \\
& e_{21}+e_{22}+e^{\prime}{ }_{21}+e_{22}^{\prime}-e_{23}^{\prime}-e_{24}^{\prime}=i_{2} r_{2}+0,5 u_{L}, \\
& e_{23}+e_{24}-e_{21}^{\prime}-e_{22}^{\prime}+e_{23}^{\prime}+e_{24}^{\prime}=-i_{2} r_{2}+0,5 u_{L},
\end{aligned}
$$




$$
\begin{aligned}
& \Sigma F_{\mathrm{I}}=i_{11} w_{1}+I_{0} w_{0}+i_{21} w_{2}+i_{2} w_{2}^{\prime}=\oint_{l_{\mathrm{I}}} H_{\mathrm{I}} d l, \\
& \Sigma F_{\mathrm{II}}=-i_{11} w_{1}+I_{0} w_{0}+i_{21} w_{2}+i_{2} w_{2}^{\prime}=\oint_{l_{\mathrm{II}}} H_{\mathrm{II}} d l, \\
& \Sigma F_{\mathrm{III}}=i_{12} w_{1}+I_{0} w_{0}+i_{22} w_{2}-i_{2} w_{2}^{\prime}=\oint_{l_{\mathrm{III}}} H_{\mathrm{III}} d l, \\
& \Sigma F_{\mathrm{IV}}=-i_{12} w_{1}+I_{0} w_{0}+i_{22} w_{2}-i_{2} w_{2}^{\prime}=\oint_{i_{\mathrm{IV}}} H_{\mathrm{IV}} d l, \\
& \Sigma F_{L}=i_{21}+i_{22}=\oint_{l} H d l, \\
& i_{2}=i_{21}-i_{22},
\end{aligned}
$$

причем $e_{21}=K_{2} e_{11}, \quad e_{22}=K_{2} e_{12}, \quad e_{23}=K_{2} e_{13}, \quad e_{24}=K_{2} e_{14}, \quad e_{21}^{\prime}=K_{2}^{\prime} e_{11}, \quad e_{22}^{\prime}=$ $=K^{\prime}{ }_{2} e_{12}, e^{\prime}{ }_{23}=K^{\prime}{ }_{2} e_{13}, e^{\prime}{ }_{24}=K^{\prime}{ }_{2} e_{14} \quad$ и коэффициенты трансформации $K_{2}=w_{2} / w_{1}, \quad K_{2}^{\prime}=w_{2}^{\prime} / w_{1} \quad$ и $\quad K_{0}=w_{0} / w_{1}$.

При решении исходной системы уравнений для отдельных состояний ДМУЧ имеем в виду, что в силу принятых предположений вентили $B_{1}$ и $B_{2}$ на рис. 1 входят в контур, содержащий только источники э. д. с. и индуктивность дросселя $L$. Согласно определению $\left[{ }^{5}\right]$, они относятся к вентилям первой группы, которые по отношению к переменной составляющей тока можно заменить проводниками, поскольку при обходе этого контура все вентили имеют одинаковое направление. При этом работа получившейся схемы будет отличаться от работы исходной схемы лишь появлением в контуре в установившемся режиме постоянной составляющей тока, равной максимальному значению тока в контуре при закороченных вентилях. Это вытекает из анализа процесса включения $r L$-цепи, содержащей идеальный вентиль, на периодическое напрлжение при $r \rightarrow 0$.

Таким образом,

$$
i_{21}=i_{21-}+i_{21 \sim}
$$

и

$$
i_{22}=i_{22-}+i_{22 \sim},
$$

где $i_{21-}$ и $i_{22-}$ - постоянные составляющие токов в выходных цепях первого и второго элементарных МУЧ (рис. 1); $i_{21} \sim$ и $i_{22 \sim}-$ переменные составляющие соответствующих токов.

Согласно вышесказанному и выбранному направлению вентилей,

$$
i_{21-}=i_{22-}=\frac{1}{2} I_{21 m}=\frac{1}{2} I_{22 m}
$$

где $I_{21 m}$ и $I_{22 m}$ - максимальные значения переменных составляющих тока в параллельных ветвях выходной цепи. имеем

Для переменных составляющих по первому закону Кирхгофа

$$
i_{21 \sim}=-i_{22 \sim}=\frac{1}{2} i_{2}
$$




\begin{tabular}{|c|c|c|}
\hline & \multicolumn{2}{|c|}{ Состояние } \\
\hline & 0110 & 1010 \\
\hline
\end{tabular}

$e_{11}-U_{1 m} \sin \omega t$

0

$e_{12} 0$

$U_{1 m} \sin \omega t$

$e_{13} 0$

0

$e_{14} U_{1 m} \cos \omega t$

$U_{1 m} \cos \omega t$

$u_{L} \sqrt{2} U_{1 m} K_{2} \cos \left(\omega t+\frac{\pi}{4}\right)$

$\sqrt{2} U_{1 m} K_{2} \sin \left(\omega t+\frac{\pi}{4}\right)$

$i_{11}-\frac{\sqrt{2}}{4} \cdot \frac{U_{1 m}}{r_{2}} K\left[K_{2}-K \sin \left(\omega t+\frac{\pi}{4}\right)\right]-K_{0} I_{0}$

$\frac{\sqrt{2}}{4} \frac{U_{1 m}}{r_{2}} K\left[K_{2}-K \cos \left(\omega t+\frac{\pi}{4}\right)\right]+K_{0} I_{0}$

$i_{12} \frac{\sqrt{2}}{4} \frac{U_{1 m}}{r_{2}} K\left[K_{2}+K \sin \left(\omega t+\frac{\pi}{4}\right)\right]+K_{0} I_{0}$

$\frac{\sqrt{2}}{4} \frac{U_{1 m}}{r_{2}} K\left[K_{2}+K \cos \left(\omega t+\frac{\pi}{4}\right)\right]+K_{0} I_{0}$

$i_{21} \frac{\sqrt{2}}{4} \frac{U_{1 m}}{r_{2}} K\left[1-\sin \left(\omega t+\frac{\pi}{4}\right)\right]$

$\frac{\sqrt{2}}{4} \frac{U_{1 m}}{r_{2}} K\left[1-\cos \left(\omega t+\frac{\pi}{4}\right)\right]$

$i_{22} \frac{\sqrt{2}}{4} \frac{U_{1 m}}{r_{2}} K\left[1+\sin \left(\omega t+\frac{\pi}{4}\right)\right]$

$\frac{\sqrt{2}}{4} \frac{U_{1 m}}{r_{2}} K\left[1+\cos \left(\omega t+\frac{\pi}{4}\right)\right]$

$i_{2}-\frac{\sqrt{2}}{2} \frac{U_{1 m}}{r_{2}} K \sin \left(\omega t+\frac{\pi}{4}\right)$

$-\frac{\sqrt{2}}{2} \frac{U_{1 m}}{r_{2}} K \cos \left(\omega t+\frac{\pi}{4}\right)$

Учитывая, что для ненасыщенного сердечника $\Sigma F=\oint_{l} H d l=0$ и для насыщенного $e=-d \Psi / d t=0$, и принимая $K=K_{2}+2 K_{2}{ }^{\prime}$, из решения системы уравнений (1) с учетом табл. 1 получим приведенные в табл. 2 и 3 уравнения напряжений, э. д. с. и токов для различных состояний ДМУЧ.

На основе полученных выражений на рис. 2-5 показаны кривые э. д. с., напряжений и токов, а также кривые изменения магнитной индукции в сердечниках ДМУЧ.

При $r_{2} \geqslant r_{2 к р}$ ДМУЧ работает в режиме б, причем состояния сердечников меняются через каждый полупериод напряжения питания (рис. 2). В этом режиме постоянные составляющие токов параллельных ветвей имеют вид

$$
i_{21-}=i_{22-}=\frac{1}{2}\left|I_{2 m}\right|=\frac{\sqrt{2}}{4} K \frac{U_{1 m}}{r_{2}}
$$


0

$$
\begin{array}{ll}
-\sqrt{2} U_{1 m} K_{2} \cos \left(\omega t+\frac{\pi}{4}\right) & -\sqrt{2} U_{1 m} K_{2} \sin \left(\omega t+\frac{\pi}{4}\right) \\
\frac{\sqrt{2}}{4} \cdot \frac{U_{1 m}}{r_{2}} K\left[K_{2}+K \sin \left(\omega t+\frac{\pi}{4}\right)\right]+K_{0} I_{0} & -\frac{\sqrt{2}}{4} \cdot \frac{U_{1 m}}{r_{2}} K\left[K_{2}+K \cos \left(\omega t+\frac{\pi}{4}\right)\right]-K_{0} I_{0} \\
-\frac{\sqrt{2}}{4} \frac{U_{1 m}}{r_{2}} K\left[K_{2}-K \sin \left(\omega t+\frac{\pi}{4}\right)\right]-K_{0} I_{0} & -\frac{\sqrt{2}}{4} \cdot \frac{U_{1 m}}{r_{2}} K\left[K_{2}-K \cos \left(\omega t+\frac{\pi}{4}\right)\right]-K_{0} I_{0} \\
\frac{\sqrt{2}}{4} \frac{U_{1 m}}{r_{2}} K\left[1+\sin \left(\omega t+\frac{\pi}{4}\right)\right] & \frac{\sqrt{2}}{4} \frac{U_{1 m}}{r_{2}} K\left[1+\cos \left(\omega t+\frac{\pi}{4}\right)\right] \\
\frac{\sqrt{2}}{4} \frac{U_{1 m}}{r_{2}} K\left[1-\sin \left(\omega t+\frac{\pi}{4}\right)\right] & \frac{\sqrt{2}}{4} \frac{U_{1 m}}{r_{2}} K\left[1-\cos \left(\omega t+\frac{\pi}{4}\right)\right] \\
\frac{\sqrt{2}}{2} \frac{U_{1 m}}{r} K \sin \left(\omega t+\frac{\pi}{4}\right) & \frac{\sqrt{2}}{2} \frac{U_{1 m}}{r_{2}} K \cos \left(\omega t+\frac{\pi}{4}\right)
\end{array}
$$

где $I_{2 m}$ - максимальное значение выходного тока. Для токов первичных цепей $i_{11}$ и $i_{12}$, а также для параллельных ветвей $i_{21}$ и $i_{22}$ в этом режиме справедливы формулы в табл. 2.

Если же $r_{2}<r_{2 \text { кр }}$ то в работе ДМУЧ наряду с режимом $б$ возникает и режим $a$ (рис. 3 ), причем длительность обоих режимов при неизменном токе подмагничивания зависит от величины сопротивления нагрузки $r_{2}$. Так как в режиме $a$, согласно соотношению (4) и табл. 3 ,

$$
i_{21-}=i_{22-}=\frac{K_{0}}{2 K_{2}^{\prime}} I_{0} \text {, }
$$

то выражения токов параллельных ветвей и первичного тока для режима б несколько меняются и принимают вид, показанный в табл. 4.

Для определения граничных значений $r_{2}$, сөответствующих переходам от одного режима к другому, рассмотрим работу ДМУЧ при $r_{2 \text { кр }}^{\prime} \leqslant r_{2} \leqslant r_{2 \text { кр. }}$ В этом случае режим $a$ существует в интервале 


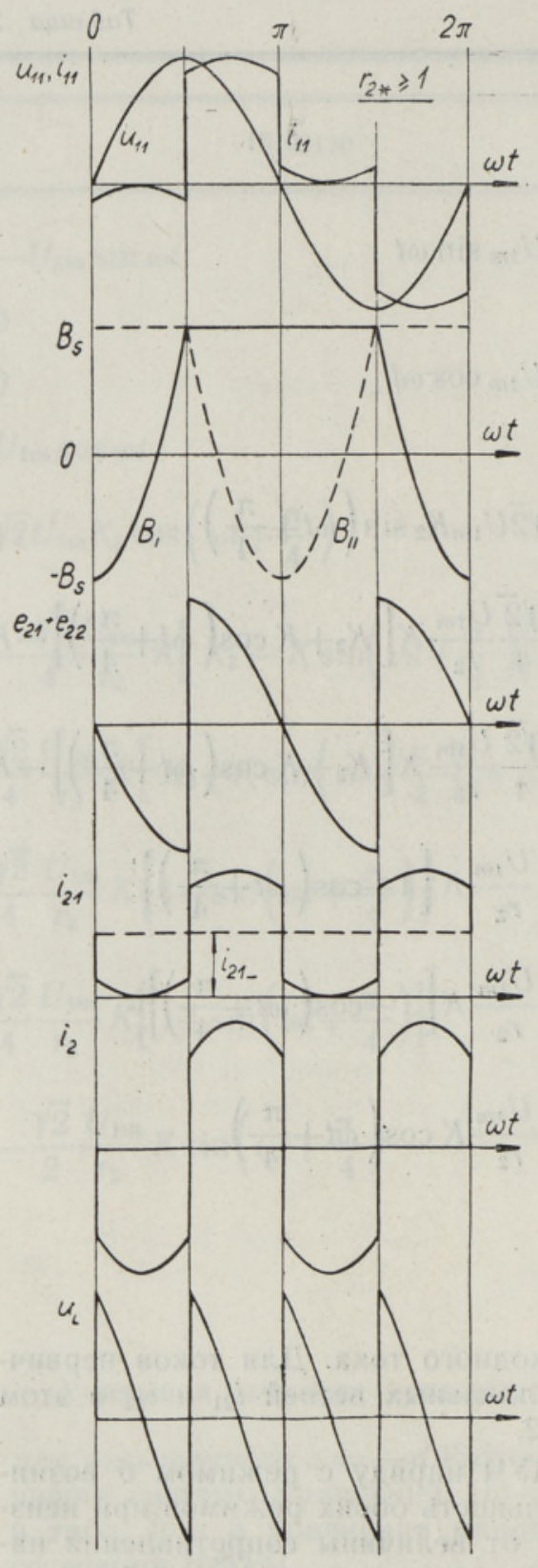

Рис. 2.

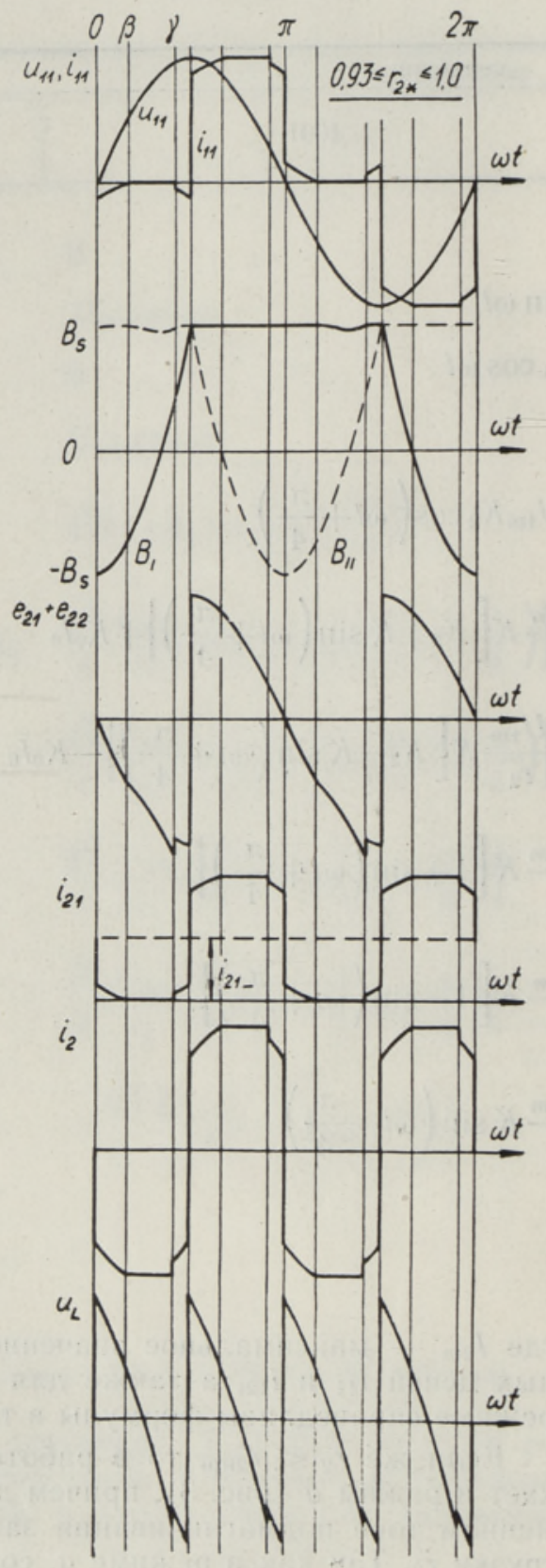

Рис. 3.

$\beta \leqslant \omega t \leqslant \gamma$ (рис. 3 ). Зависимость угла $\beta$, соответствующего моменту обращения тока $i_{11}$ в нуль, от параметров схемы определяется из уравнения для $i_{11}$ в состоянии 0110 в табл. 4 подстановкой $\omega t=\beta$ :

$$
\beta=\arcsin \frac{\sqrt{2} K_{0}}{K K_{2}^{\prime}} \frac{I_{0} r_{2}}{U_{1 m}}-\frac{\pi}{4} .
$$


Зависимость угла $\gamma$, соответствующего моменту окончания режима $a$, от параметров схемы следует из условия, что среднее за период значение э. д. с. одного сердечника должно равняться нулю. Например, для первого сердечника

$$
\frac{1}{2 \pi} \int_{\beta}^{2 \pi+\beta} e_{11} d \omega t=0
$$

откуда

$$
\cos \left(\gamma+\frac{\pi}{4}\right)-\cos \left(\beta+\frac{\pi}{4}\right)=(\beta-\gamma) \sin \left(\beta+\frac{\pi}{4}\right) .
$$

С увеличением $r_{2}$ продолжительность режима $a$ сокращается и на-

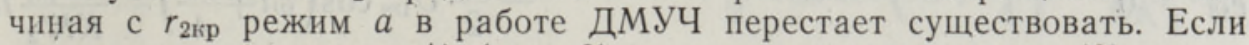
$r_{2}=r_{2 \kappa р}$, то $\beta=\gamma=\pi / 4$ (рис. 2) и, согласно выражению (8),

$$
r_{2 \kappa \mathrm{p}}=\frac{\sqrt{2}}{2} \frac{K K_{2}^{\prime}}{K_{0}} \frac{U_{1 m}}{I_{0}}
$$

С уменьшением $r_{2}$ угол $\gamma$ увеличивается и при некотором значении $r_{2}=r_{2 к р}^{\prime}$ будет $\gamma=\pi / 2$ и $\beta=\beta^{\prime}$. Подстановкой этих значений в формулу (10) получим трансцендентное уравнение для определения $\boldsymbol{\beta}^{\prime}$ :

$$
\frac{\sqrt{2}}{2}+\cos \left(\beta^{\prime}+\frac{\pi}{4}\right)=\left(\frac{\pi}{2}-\beta^{\prime}\right) \sin \left(\beta^{\prime}+\frac{\pi}{4}\right),
$$

откуда $\beta^{\prime}=22^{\circ} 41^{\prime}$. Соответствующее граничное сопротивление нагрузки вытекает из (8):

$$
r_{2 \kappa p}^{\prime}=\frac{\sqrt{2}}{2} \frac{K K_{2}^{\prime}}{K_{0}} \frac{U_{1 m}}{I_{0}} \sin \left(\beta^{\prime}+\frac{\pi}{4}\right) .
$$

Для случая $r_{2 \text { кр }} \leqslant r_{2 \text { кр }} \leqslant r_{2 \text { п }}$ чередование состояний сердечников определяется углами $\alpha$ и $\beta$ (рис. 4), причем зависимость $\alpha=f(\beta)$ получается так же, как и зависимость угла $\gamma$ :

$$
\cos \left(\alpha+\frac{\pi}{4}\right)+\cos \left(\beta+\frac{\pi}{4}\right)=\left(\frac{\pi}{2}+\alpha-\beta\right) \sin \left(\beta+\frac{\pi}{4}\right) .
$$

С уменьшением $r_{2}$ угол $\alpha$ увеличивается и угол $\beta$ уменьшается так, что при некотором значении $r_{2}=r_{2 \pi} \alpha=\beta=\alpha_{\text {II }}$ и ток нагрузки принимает прямоугольную форму (рис. 5). Подставив $\alpha=\beta=\alpha_{\text {II }}$ в формулу (14), получим трансцендентное уравнение для определения граничного угла $\alpha_{\Pi}$ :

$$
\alpha_{\mathrm{II}}=\operatorname{arctg} \frac{4}{\pi}-\frac{\pi}{4}=6^{\circ} 51^{\prime} .
$$

Соответствующее сопротивление нагрузки:

$$
r_{2 \Pi}=\frac{\sqrt{2}}{2} \frac{K K_{2}^{\prime}}{K_{0}} \frac{U_{1 m}}{I_{0}} \sin \left(\alpha_{\Pi}+\frac{\pi}{4}\right) .
$$


$e_{11} \quad \frac{\sqrt{2}}{2} U_{1 m} \cos \left(\omega t+\frac{\pi}{4}\right)-\frac{K_{0}}{K K_{2}^{\prime}} I_{0} r_{2} \quad 0$

$e_{12} \quad \frac{\sqrt{2}}{2} U_{1 m} \sin \left(\omega t+\frac{\pi}{4}\right)-\frac{K_{0}}{K K_{2}^{\prime}} I_{0} r_{2} \quad U_{1 m} \sin \omega t$

$e_{13} \quad 0$

$-\frac{\sqrt{2}}{2} U_{1 m} \cos \left(\omega t+\frac{\pi}{4}\right)-\frac{K_{0}}{K K_{2}^{\prime}} I_{0} r_{2}$

$e_{14} \quad U_{1 m} \cos \omega t$

$\frac{\sqrt{2}}{2} U_{1 m} \sin \left(\omega t+\frac{\pi}{4}\right)-\frac{K_{0}}{K K_{2}^{\prime}} I_{0} r_{2}$

$u_{L} \quad 2 K_{2} U_{1 m} \cos \omega t-2 \frac{K_{2} K_{0}}{K K_{2}^{\prime}} I_{0} r_{2}$

$2 K_{2} U_{1 m} \sin \omega t-2 \cdot \frac{K_{2} K_{0}}{K K_{2}^{\prime}} I_{0} r_{2}$

$i_{11} \quad 0$

$i_{12} \quad \frac{K K_{0}}{K^{\prime}{ }_{2}} I_{0}$

$\frac{K K_{0}}{K^{\prime}{ }_{2}} I_{0}$

$i_{21} \quad 0$

$i_{22} \quad \frac{K_{0}}{K_{2}^{\prime}} I_{0}$

0

$i_{2} \quad-\frac{K_{0}}{K^{\prime}{ }_{2}} I_{0}$

$\frac{K_{0}}{K_{2}^{\prime}} I_{0}$

0

$\frac{K_{0}}{K_{2}^{\prime}} I_{0}$

$i_{11} \frac{\sqrt{2}}{4} \frac{U_{1 m}}{r_{2}} K^{2} \sin \left(\omega t+\frac{\pi}{4}\right)-\frac{K_{0} K}{2 K_{2}^{\prime}} I_{0} \quad-\frac{\sqrt{2}}{4} \frac{U_{1 m}}{r_{2}} K^{2} \cos \left(\omega t+\frac{\pi}{4}\right)+\frac{K_{0} K}{2 K_{2}^{\prime}} I_{0}$

$i_{12} \frac{\sqrt{2}}{4} \frac{U_{1 m}}{r_{2}} K^{2} \sin \left(\omega t+\frac{\pi}{4}\right)+\frac{K_{0} K}{2 K_{2}^{\prime}} I_{0} \quad \frac{\sqrt{2}}{4} \frac{U_{1 m}}{r_{2}} K^{2} \cos \left(\omega t+\frac{\pi}{4}\right)+\frac{K_{0} K}{2 K_{2}^{\prime}} I_{0}$

$i_{21}-\frac{\sqrt{2}}{4} \frac{U_{1 m}}{r_{2}} K \sin \left(\omega t+\frac{\pi}{4}\right)+\frac{K_{0}}{2 K_{2}^{\prime}} I_{0} \quad-\frac{\sqrt{2}}{4} \frac{U_{1 m}}{r_{2}} K \cos \left(\omega t+\frac{\pi}{4}\right)+\frac{K_{0}}{2 K_{2}^{\prime}} I_{0}$

$i_{22} \frac{\sqrt{2}}{4} \frac{U_{1 m}}{r_{2}} K \sin \left(\omega t+\frac{\pi}{4}\right)+\frac{K_{0}}{2 K_{2}^{\prime}} I_{0} \quad \frac{\sqrt{2}}{4} \frac{U_{1 m}}{r_{2}} K \cos \left(\omega t+\frac{\pi}{4}\right)+\frac{K_{0}}{2 K_{2}^{\prime}} I_{0}$ 
Таблица 3

сердечников

0001

.0100

$-\frac{\sqrt{2}}{2} U_{1 m} \sin \left(\omega t+\frac{\pi}{4}\right)-\frac{K_{0}}{K K_{2}^{\prime}} I_{0} r$

$-U_{1 m} \sin \omega t$

$-\frac{\sqrt{2}}{2} U_{1 m} \cos \left(\omega t+\frac{\pi}{4}\right)-\frac{K_{0}}{K K_{2}^{\prime}} I_{0} r_{2}$

0

$-U_{1 m} \cos \omega t$

$-\frac{\sqrt{2}}{2} U_{1 m} \sin \left(\omega t+\frac{\pi}{4}\right)-\frac{K_{0}}{K K_{2}^{\prime}} I_{0} r_{2}$

$\frac{\sqrt{2}}{2} U_{1 m} \cos \left(\omega t+\frac{\pi}{4}\right)-\frac{K_{0}}{K K_{2}^{\prime}} I_{0} r_{2}$

$-2 K_{2} U_{1 m} \cos \omega t-2 \frac{K_{2} K_{0}}{K K_{2}^{\prime}} I_{0} r_{2}$

$-2 K_{2} U_{1 m} \sin \omega t-2 \cdot \frac{K_{2} K_{0}}{K K_{2}^{\prime}} I_{0} r_{2}$

$-\frac{K K_{0}}{K_{2}^{\prime}} I_{0}$

U

0

$\frac{K_{0}}{K_{2}^{\prime}} I_{0}$

0

$\frac{K_{0}}{K_{2}^{\prime}} I_{0}$

Таблица 4

$\frac{\sqrt{2}}{4} \frac{U_{1 m}}{r_{2}} K^{2} \sin \left(\omega t+\frac{\pi}{4}\right)+\frac{K_{0} K}{2 K_{2}^{\prime}} I_{0}$

$-\frac{\sqrt{2}}{4} \frac{U_{1 m}}{r_{2}} K^{2} \cos \left(\omega t+\frac{\pi}{4}\right)-\frac{K_{0} K}{2 K_{2}^{\prime}} I_{0}$

$\frac{\sqrt{2}}{4} \frac{U_{1 m}}{r_{2}} K^{2} \sin \left(\omega t+\frac{\pi}{4}\right)-\frac{K_{0} K}{2 K_{2}^{\prime}} I_{0}$

$\frac{\sqrt{2}}{4} \frac{U_{1 m}}{r_{2}} K^{2} \cos \left(\omega t+\frac{\pi}{4}\right)-\frac{K_{0} K}{2 K_{2}^{\prime}} I_{0}$

$\frac{\sqrt{2}}{4} \frac{U_{1 m}}{r_{2}} K \sin \left(\omega t+\frac{\pi}{4}\right)+\frac{K_{0}}{2 K^{\prime}} I_{0}$

$\frac{\sqrt{2}}{4} \frac{U_{1 m}}{r_{2}} K \cos \left(\omega t+\frac{\pi}{4}\right)+\frac{K_{0}}{2 K^{\prime}} I_{0}$

$-\frac{\sqrt{2}}{4} \frac{U_{1 m}}{r_{2}} K \sin \left(\omega t+\frac{\pi}{4}\right)+\frac{K_{0}}{2 K_{2}^{\prime}} I_{0}$

$-\frac{\sqrt{2}}{4} \frac{U_{1 m}}{r_{2}} K \cos \left(\omega t+\frac{\pi}{4}\right)+\frac{K_{0}}{2 K_{2}^{\prime}} I_{0}$ 


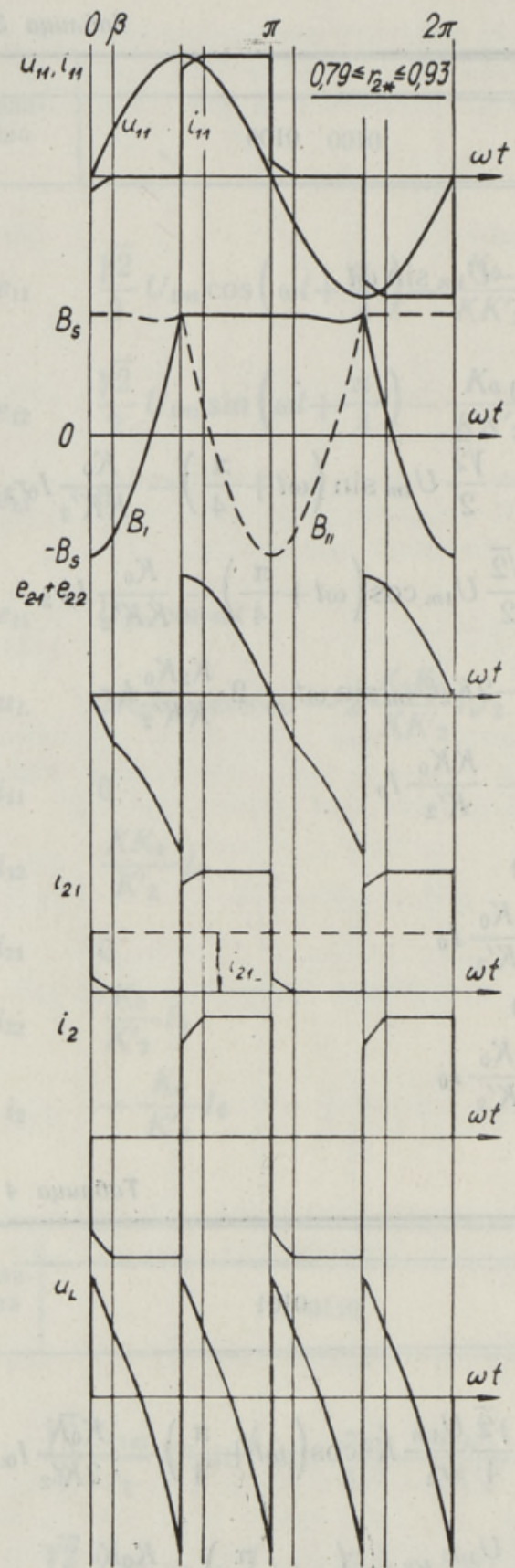

Рис. 4.

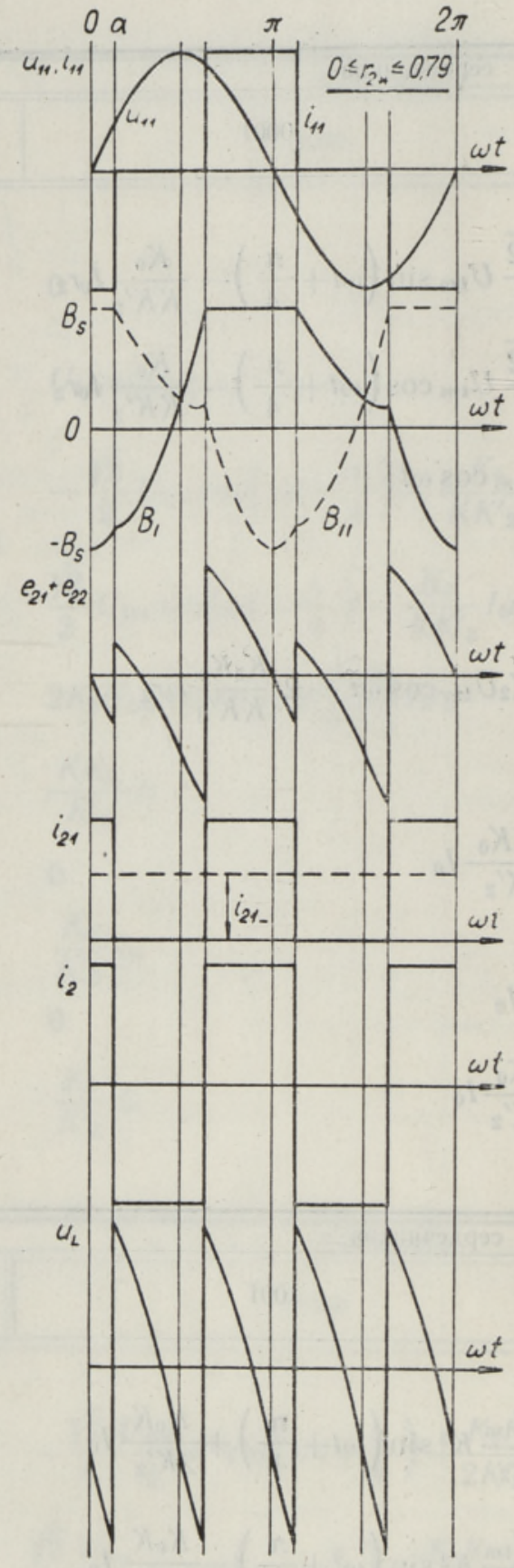

Рис. 5.

При дальнейшем уменьшении $r_{2}$ в диапазоне $r_{2 \pi} \leqslant r_{2} \leqslant 0$ прямоугольная форма тока нагрузки сохраняется. Согласно условию, что среднее за период значение э. д. с. одного сердечника должно равняться нулю, зависимость угла $\alpha$ от параметров схемы описывастся выражением 


$$
\alpha=\arccos \cdot \frac{\sqrt{2} \pi}{4} \frac{K_{0}}{K K_{2}^{\prime}} \frac{I_{0} r_{2}}{U_{1 m}}-\frac{\pi}{4} .
$$

В граничном случае при $r_{2}=0$ имеем $\alpha=\pi / 4$.

Таким образом, в ДМУЧ в зависимости от величины сопротивления нагрузки можно выделить четыре разных режима работы, отличающиеся наличием и чередованием во времени насыщенных и ненасыщенных состояний сердечников. Выведенные для этих режимов уравнения служат основой для расчета внешних характеристик и характеристик управления ДМУЧ.

\section{Л И Т Е Р Т У Р А}

1. С а к к ос Т., Изв. АН ЭССР, Физ. Матем., 24, 63 (1975).

2. С аккос Т. Ю., В сб.: Цепи преобразования параметров электроэнергии, Таллин, 1975 , с. 113.

3. С ар в В., О яв ээ р М., С аккос Т., Х ун т Ю., В сб.: Магнитные преобразователи электроэнергии с удвоением частоты, Таллин, 1972, с. 368.

4. Л ау см а а Т. М., Пя рн Р. Я., С аккос Т. Ю., С а р в В. В., Регулируемый удвоитель частоты, Авт. свид. СССР № 524293, Бюл. изобр., 1976, № 29.

5. Д ан ил о в Л. В., Электричество, № 5, 91 (1967).

Ннститут термофизики и электрофизики Академии наук Эстонской ССР

Поступила в редакцию $31 / \mathrm{V} 1976$

\section{Tiiu SAKKOS}

\section{STATSIONAARSED PROTSESSID TRANSFORMATOORSES KAHEKORDISTATUD SAGEDUSEGA VAHELDUVVOOLUREGULAATORIS}

Artiklis on analüüsitud täiustatud transformatoorse vahelduvvooluregulaatori tööd eeldusel, et südamike magneetimiskõver on täisnurkne. On tuletatud valemid üksikute ahelate emj., voolude ja pingete arvutamiseks. Teoreetilised tulemused on kooskőlas katseandmetega.

\section{Tiiu SAKKOS}

\section{STATIONARY PROCESSES IN TRANSFORMER-COUPLED ALTERNATIVE CURRENT REGULATOR}

The author analyzes the operation of the improved transformer-coupled ac regulator in the case of rectangular magnetization curve of the cores. Expressions of electromotive forces, currents and voltages of single circuits are derived. 